\title{
A EFETIVIDADE DAS ESTRATÉGIAS DE ADVERGAMING: UM ESTUDO EXPERIMENTAL COMPARANDO ADVERGAMES E IN-GAME ADVERTISINGS
}

Objetivo: Este artigo objetivou explicitar o efeito gerado por estratégias de advergaming na atitude dos jogadores em relação à marca, assim como a frequência de lembrança e recordação proporcionadas.

Método: Foram comparadas as características das principais estratégias do advergaming, os advergames (jogos com temática de marcas) e os in-game advertisings (jogos com inserção de marcas). Para testar as hipóteses de pesquisa foi realizado experimento com amostra de 165 estudantes universitários, dividida de forma randômica em três grupos que jogaram jogos criados na plataforma GameSalad para o estudo, com diferentes níveis de exposição de marca: jogo sem inserção de marca, jogo com inserção da marca fictícia no background (in-game advertising) e jogo com temática da marca fictícia (advergame).

Originalidade/Relevância: Tendo em vista o escasso número de trabalhos sobre o tema advergaming, sobretudo em periódicos nacionais onde ainda não foram publicados trabalhos realizando experimentoa acerca de sua efetividade, este artigo contribui para melhor compreensão do tema, apresentando uma reflexão sobre a efetividade dos in-game advertisings e advergames.

Resultados: Os resultados mostraram que advergames e in-game advertisings provocam uma mudança significativamente positiva na atitude em relação à marca e que as frequências observadas de lembrança e recordação da marca são significativamente maiores em advergames em comparação a in-game advertisings.

Contribuições teóricas/metodológicas: $\mathrm{O}$ artigo apresenta evidências de que a utilização de advergaming é efetiva na construção de uma atitude favorável em relação à marca, assim como no aspecto da construção da conscientização de marca.

Palavras-chave: Advergaming. Advergame. In-game Advertising. Estratégia Promocional Interativa. Internet.

\section{THE EFFECTIVENESS OF ADVERGAMING STRATEGIES: AN EXPERIMENTAL STUDY COMPARING ADVERGAMES AND IN-GAME ADVERTISINGS}

Objective: This article aimed to explain the generated effect of advergaming strategies in the attitude of the players towards the brand, as well as the recall of the brand.

Method: Were compared the characteristics of the main strategies of advergaming, the advergames (brand-themed games) and the in-game advertisings (games with the addition of brands). To test the hypotheses an experiment was conducted with a sample of 165 University students, divided randomly into three groups that played games created in GameSalad platform with different exposure levels of brand: game without brand, fictional brand insertion in the background (in-game advertising) and fictional brand-themed game (advergaming).

Originality/Relevance: Considering the small number of papers on the advergaming theme, especially in national journals where no studies have yet been published experimenting its effectiveness, this article contributes to a better understanding of the theme, presenting a reflection on the effectiveness of in-game advertisings and advergames.

Results: The results showed that advergames and in-game advertisings cause a significantly positive change in attitude towards the brand and that the observed frequency of memory and recall of the brand are significantly higher in advergames compared to in-game advertisings.

Theoretical/Methodological Contributions: The paper presents evidence that the use of advergaming is effective in building a favorable attitude towards the brand, as well as in the aspect of building brand awareness.

Keywords: Advergaming. Advergame. In-game Advertising. Interactive Promotional Strategy. Internet.

Izabella Bueno Fernandes ${ }^{1}$ Ricardo Teixeira Veiga ${ }^{2}$ Fábio Roberto Ferreira Borges ${ }^{3}$

\footnotetext{
${ }^{1}$ Mestre em Administração pela Universidade Federal de Minas Gerais - UFMG. Professora no Centro Universitário de Belo Horizonte UNI-BH. Minas Gerais, Brasil. E-mail: izabellafernandes@ hotmail.com

${ }^{2}$ Doutor em Administração pela Universidade Federal de Minas Gerais - UFMG. Professor da Universidade Federal de Minas Gerais - UFMG. Minas Gerais, Brasil. E-mail: ricardonecc@gmail.com

${ }^{3}$ Doutor em Administração pela Universidade Federal de Minas Gerais - UFMG. Minas Gerais, Brasil. E-mail: fabiorfborges@gmail.com
} 


\section{INTRODUÇÃO}

O advento da web 2.0 transformou a internet em um canal multidirecional, alterando profundamente a maneira como se relacionam empresas e consumidores (JAFFE, 2008). Neste novo contexto, a interatividade se tornou primordial para que as empresas consigam promover o engajamento de seus clientes e aumentar o valor de suas marcas (TUTEN; SOLOMON, 2013). Assim, estratégias promocionais intrusivas e interruptivas convivem com novas abordagens, que conseguem usufruir dos espaços de participação da web. Dentre essas novas abordagens, a inserção de marcas em jogos digitais se destaca pela capacidade destes jogos de entreter de forma interativa enquanto é transmitida uma mensagem de marketing.

Somente no Brasil, entre jogadores casuais e aficionados, 11,8 milhões de pessoas possuem o hábito de se entreterem com jogos digitais, em uma indústria que movimenta quase um bilhão de reais por ano Instituto Brasileiro de Opinião Pública e Estatística [IBOPE, 2012]. De acordo com pesquisa realizada pela Newzoo (2016), são esperados US $\$ 108,9$ bilhões em receitas de jogos em 2017, representando um aumento de US\$7,8 bilhões em relação ao ano de 2016. Estima-se que no ano de 2020 serão gerados US $\$ 128,5$ bilhões em receitas, demonstrando o potencial de crescimento do setor (NEWZOO, 2016). Sendo assim, em um ambiente em que as empresas necessitam de novas abordagens interativas para se aproximarem dos consumidores, o advergaming, ou seja, a entrega de mensagens de marketing em jogos digitais (HERNANDEZ et al., 2004), assumiu papel de destaque nas estratégias promocionais.

O Brasil representa o maior mercado na América Latina, gerando US $\$ 1,3$ bilhões em receita no mercado de jogos (NEWZOO, 2016). Com um público tão grande consumindo e interagindo com jogos digitais, as empresas vislumbram nesta forma de entretenimento um canal para a exposição de suas mensagens, buscando promover engajamento de uma forma não intrusiva (TUTEN; SOLOMON, 2013). Sendo assim, compreendendo o cenário promissor, este estudo buscou responder a seguinte pergunta: Qual é a efetividade das estratégias promocionais de marketing que utilizam jogos digitais, considerando a atitude em relação à marca e à conscientização da marca?

Este artigo teve como objetivo geral explicitar o efeito gerado por estratégias de advergaming na atitude dos jogadores em relação à marca e à efetividade dessas estratégias, assim como as frequências de lembrança e recordação proporcionadas por essa exposição.

Tendo em vista o escasso número de trabalhos sobre o tema advergaming, sobretudo em periódicos nacionais onde ainda não foram publicados quaisquer trabalhos realizando um experimento acerca de sua efetividade, este artigo contribui para melhor compreensão do tema, apresentando uma reflexão sobre a efetividade dos in-game advertisings e advergames. Além disso, pelo uso crescente da internet e das redes socais em diversos dispositivos móveis e computadores, o hábito de entretenimento via jogos digitais passa a ter maior abrangência (CAMPI, 2012), o que torna este objeto de estudo relevante, sendo necessário compreender, na perspectiva acadêmica, a percepção do consumidor em relação à inserção de marcas nestes conteúdos e contribuir gerencialmente para que as empresas possam ser mais efetivas nas iniciativas que incluem jogos digitais. Publicações científicas estão se debruçando sobre o tema à medida que a interação do consumidor com uma marca em um contexto de jogo é algo que proporciona novas experiências que afetam o comportamento do consumidor, justificando com o que se realiza com estudos para compreender a natureza dessas experiências e os efeitos proporcionados pelas mesmas (HERNANDEZ et al, 2004; CAUBERGHE, PELSMACKER, 2010; PARREÑO, PÉREZ, GARCIA, 2010).

\section{FUNDAMENTAÇÃO TEÓRICA}

\subsection{Advergaming}

Advergaming é uma ação promocional em que as empresas utilizam jogos para divulgar seus produtos, serviços e marcas (LAMB; HAIR; McDANIEL, 2010). Tais jogos possibilitam que essas empresas alcancem audiências específicas, promovam engajamento e apresentem mensagens de forma não intrusiva (TUTEN; SOLOMON, 2013). Enquanto na comunicação tradicional há um fluxo unidirecional de empresas para consumidores e os indivíduos são expostos a uma mensagem durante um curto período de tempo, nos jogos esta mensagem é interativa e pode promover maior envolvimento, dependendo apenas da qualidade do entretenimento proporcionado pelo jogo, seu tema e sua narrativa (JAFFE, 2008).

No contexto do advergaming, as empresas escolhem entre duas estratégias promocionais:

a) In-game Advertising, ou inserção de marcas em videogames - inserção de marcas em jogos produzidos sem o propósito exclusivo de divulgar uma marca específica (LENG; QUAH; ZAINUDDIN, 2010); 
b) Advergames - um jogo criado para a divulgação de uma marca específica (WISE et al., 2008).

A interatividade é um conceito essencial à relação de atitude positiva do consumidor para com a marca ou produto, pois o nível de atenção e interesse para a propaganda é, muitas vezes, insuficiente, mas com a possibilidade de entretenimento, promoção e competitividade oferecida pela estratégia de advergaming ele pode aumentar (OLIVEIRA, 2012).

\subsection{Advergame}

O advergame é um jogo desenvolvido em torno de uma marca. Representa uma forma única de entretenimento, pois, em contraste com outras formas de exposição sutil de produtos e marcas em jogos, é produzido especificamente para uma marca, com o objetivo de divulgá-la (WISE et al., 2008). O desenvolvimento de advergames busca tornar a marca mais conhecida, aumentar a atitude positiva em relação a ela, prover informações sobre um produto e diferenciar um produto em relação a seus similares (HERNANDEZ et al., 2004).

Segundo Vaz (2010), o advergame surgiu em 1983, quando a Coca-Cola pediu uma versão do jogo Space Invaders à Atari para demonstração ao pessoal de vendas em uma convenção. As naves inimigas invasoras do jogo foram substituídas pelas letras P, E, P, S, e I. Ao derrotar todas elas, o jogo exibia a frase "Coke wins". Dessa forma, segundo Oliveira (2012), as marcas entendem, por meio dessa oportunidade, que podem criar mensagens publicitárias e, ao mesmo tempo, proporcionar entretenimento aos jogadores. De acordo com Söbke e Bröker (2015) é necessário compreender a importância da comunicação nos jogos, pois a mesma proporciona uma oportunidade de implementação durante o processo de desenho do jogo, no sentido de melhorar a experiência, a fidelidade do cliente, os objetivos de marketing ou as experiências de aprendizagem.

Conforme destacam Gaarbo e Johnsen (2012), os advergames podem ser disponibilizados no website da marca, como parte da estratégia de marketing da empresa e por estarem sempre disponíveis para jogar. Atualmente, as marcas exploram o espaço mais adequado para disponibilizar advergames: as redes sociais. Nelas, o advergame, também chamado, neste caso, de social advergame, pode estimular uma disputa entre uma rede de amigos e conhecidos, apresentando o ranking das conquistas e pontuações de cada usuário, assim como permitir aos usuários comentarem entre si sobre o advergame, estimulando a promoção da marca. De acordo com Wang, Lee, Mantz e Huang
(2015), na última década a maioria dos estudos explorou os efeitos do reconhecimento da marca no product placement, porém uma possibilidade para novos estudos é explorar a lembrança da marca nos advergames, o que este artigo se propõe a realizar.

\subsection{In-game Advertising}

A prática de inserir marcas em conteúdos voltados para o entretenimento não é nova. Desde a década de 1980, quando os doces Reese's Pieces apareceram no filme ET e a Pepsi no filme De Volta Para o Futuro, vem crescendo e se tornando uma alternativa viável para atrair maior atenção do público (WISE et al., 2008).

$\mathrm{O}$ in-game advertising é a inserção de marcas e produtos em jogos produzidos por um desenvolvedor, em que o jogo não foi originalmente concebido para a exposição de uma marca específica (NELSON, 2002). O in-game advertising pode ser, por exemplo, a colocação de uma marca em placa lateral em um simulador de futebol ou em um jogo de corrida, um outdoor em um jogo que simula uma guerra em ambiente urbano ou uso de camisas com determinada marca em jogo simulador de convivência. O in-game advertising é uma forma de inserir produtos/marcas num conteúdo midiático (product/brand placement) em um jogo, em que o ambiente é mais interativo e o usuário está com sua atenção voltada para o que está sendo exibido (LENG; QUAH; ZAINUDDIN, 2010).

De acordo com Nelson (2004), jogadores avaliam positivamente as marcas em jogos quando estas adicionam realismo a eles. Além disso, jogadores que avaliam negativamente a presença de marcas em jogos digitais avaliam da mesma forma a presença de propagandas, em geral. Contudo, os ingame advertisings podem não ser tão memoráveis quanto os advergames, pois neste caso o jogo é o foco primário de atenção e o processamento da presença da marca é secundário, acionado apenas por recursos cognitivos esparsos que não estão sendo utilizados na tarefa principal relacionada ao jogo (WISE et al, 2008). Segundo estudos realizados por Wang, Lee, Mantz e Huang (2015) o nível de engajamento com o jogo é proporcional com a percepção da personalidade da marca.

\section{METODOLOGIA}

Realizou-se pesquisa causal, baseada em experimento. Segundo Malhotra (2001), a inferência causal relaciona-se com a expectativa de que uma mudança em uma variável de marketing produz mudança em outra variável. O método experimental permite que variáveis independentes sejam manipuladas e seu efeito em uma ou mais variáveis 
dependentes seja verificado, a fim de testar empiricamente hipóteses de causalidade.

A Tabela 1 apresenta as hipóteses testadas e indica quais são as referências que compõem a revisão da literatura apresentada no capítulo anterior que deram sustentação para que tais hipóteses fossem desenvolvidas.

Tabela 1 - Hipóteses de pesquisa

\begin{tabular}{|c|c|c|}
\hline & Hipóteses & Suporte Teórico \\
\hline $\mathrm{H}_{1}$ & $\begin{array}{l}\text { Advergames e in-game advertisings provocam uma } \\
\text { mudança significativamente positiva na atitude em } \\
\text { relação à marca. }\end{array}$ & $\begin{array}{l}\text { Todos os trabalhos abaixo pesquisaram a } \\
\text { efetividade de advergamings por meio de } \\
\text { experimentos relacionados á atitude em relação à } \\
\text { marca: (LAMB; HAIR; McDANIEL, 2010); } \\
\text { (TUTEN; SOLOMON, 2013); (JAFFE, 2008; } \\
\text { (LENG; QUAH; ZAINUDDIN, 2010); (WISE et } \\
\text { al., 2008); (OLIVEIRA, 2012); (PARREÑ; } \\
\text { PÉREZ; GARCIA, 2010); HERNANDEZ et al } \\
\text { (2004); (REDONDO, 2010); (CAUBERGHE; } \\
\text { PELSMACKER, 2010) }\end{array}$ \\
\hline $\mathrm{H}_{2}$ & $\begin{array}{l}\text { Advergames provocam maior efeito na mudança } \\
\text { significativamente positiva na atitude em relação à } \\
\text { marca que os in-game advertisings. }\end{array}$ & $\begin{array}{l}\text { Todos os trabalhos abaixo realizaram pesquisas } \\
\text { fazendo uma comparação entre advergames e in- } \\
\text { game advertisings:(LENG; } \\
\text { ZAINUDDIN, 2010); (WISE et al., 2008); } \\
\text { (OLIVEIRA, 2012); (PARREÑO; PÉREZ; } \\
\text { GARCIA, 2010); HERNANDEZ et al (2004); } \\
\text { (REDONDO, 2010); (CAUBERGHE; } \\
\text { PELSMACKER, 2010) }\end{array}$ \\
\hline $\mathrm{H}_{3}$ & $\begin{array}{l}\text { As frequências observadas de lembrança e recordação } \\
\text { da marca presente no jogo são significativamente } \\
\text { maiores em advergames que em in-game } \\
\text { advertisings. }\end{array}$ & $\begin{array}{l}\text { Os trabalhos abaixo realizaram pesquisas fazendo } \\
\text { uma comparação entre advergames e in-game } \\
\text { advertisings relacionados a frequência de } \\
\text { recordação e lembrança da marca:(LENG; } \\
\text { QUAH; ZAINUDDIN, 2010); (CAUBERGHE; } \\
\text { PELSMACKER, 2010) }\end{array}$ \\
\hline
\end{tabular}

Fonte: Elaborado pelos autores.

Para melhor compreensão do experimento, segue a notação de seu design:

Em que:

$$
\begin{array}{lllll}
\mathrm{GC}-\mathrm{R} & \mathrm{OM}_{1} & \mathrm{X}_{1} & \mathrm{OM}_{2} & \mathrm{OL}_{1} \\
\mathrm{GE}_{1}-\mathrm{R} & \mathrm{OM}_{3} & \mathrm{X}_{2} & \mathrm{OM}_{4} & \mathrm{OL}_{2} \\
\mathrm{GE}_{2}-\mathrm{R} & \mathrm{OM}_{5} & \mathrm{X}_{3} & \mathrm{OM}_{6} & \mathrm{OL}_{3}
\end{array}
$$

GC - Grupo Controle

$\mathrm{GE}_{1}$ - Grupo Experimental 1 (in-game advertising)

$\mathrm{GE}_{2}$ - Grupo Experimental 2 (advergame)

$\mathrm{R}$ - Aleatorização

$\mathrm{X}_{1}$ - Jogo sem inserção de marca

$\mathrm{X}_{2}$ - Jogo com inserção de marca no background (in-Game advertising)

$\mathrm{X}_{3}$ - Jogo com temática de marca (advergame)

OM - Observação da atitude em relação à marca

OL - Observação da lembrança e recordação 


\subsection{Variáveis independentes}

De acordo com o objetivo geral do estudo de explicitar o efeito gerado por estratégias de advergaming na atitude dos jogadores em relação à efetividade dessas estratégias, assim como a frequência de lembrança e recordação proporcionadas por esta exposição fez-se necessária à criação de um jogo com três diferentes versões: jogo sem inserção de marca, jogo com inserção de marca no background (in-game advertising) e jogo com temática de marca (advergame).

O jogo foi desenvolvido pelos pesquisadores, durante 6 meses, a partir da plataforma para a criação de jogos GameSalad e de um template pago da DeepBlue, produtora de jogos eletrônicos, tendo um custo total de U\$25 doláres, financiado pelos próprios pesquisadores. O jogo se enquadra nas categorias de puzzle, que enfatizam solução de problemas, e arcade, que testam reflexos dos usuários, exigindo pouca habilidade estratégica ou de raciocínio complexo. O objetivo do jogador é acertar um alvo com um personagem lançado a partir de um canhão. A crescente dificuldade se dá a partir do maior número de obstáculos e elementos sujeitos às leis da física que precisam ser movidos, por exemplo, plataformas móveis. O jogo é adequado a todos os públicos e a escolha por esta categoria foi inspirada em Redondo (2010), que também utilizou um jogo da categoria puzzle.

A marca escolhida para a criação dos jogos foi fictícia, pois uma das implicações práticas do estudo realizado por Redondo (2010) é que novos estudos experimentais deveriam utilizar marcas pouco populares na construção dos jogos. Outro estudo utilizado como referência foi o de Cauberghe e Pelsmacker (2010), no qual as marcas utilizadas foram fictícias, para que fossem evitados efeitos de confusão com os conhecimentos prévios sobre elas, por parte dos participantes do estudo.

Foi criada uma marca fictícia de chocolate, categoria de produtos também utilizada por Redondo (2010), por ter apelo a todos os públicos. O nome da marca escolhido foi Chocante - Chocolate Crocante, criado com o auxílio de um profissional formado em Publicidade e Propaganda, com experiência na área de criação publicitária e branding. O logo apresentado na Figura 1 também foi criado com a ajuda deste profissional.

\section{Ch"CAnTe}

Figura 1 - Logo Chocante - Chocolate Crocante Fonte: Elaborado pelos autores.

\subsubsection{Jogo sem inserção de marca}

O jogo sem inserção de marca, chamado "The Pirate Blaster", seguia o padrão original do template. Os sujeitos experimentais do Grupo Controle interagiram com este jogo.

\subsubsection{Jogo com inserção de marca no background (in-game advertising)}

$O$ jogo com inserção de marca no background, representando a ação de in-game advertising, chamado "Pirate Blaster", seguia o padrão original do template comprado com a modificação do background do gameplay, onde foi inserida a marca Chocante - Chocolate Crocante. A inserção foi contextual, sendo que a marca foi colocada como um dos tesouros presentes no mapa representado no background do jogo. Essa inserção contextual no background foi inspirada nos trabalhos de Redondo (2010) e Cauberghe e Pelsmacker (2010). Os sujeitos experimentais do Grupo Experimental 1 interagiram com este jogo. A Figura 2 mostra os elementos gráficos do jogo Pirate Blaster. 


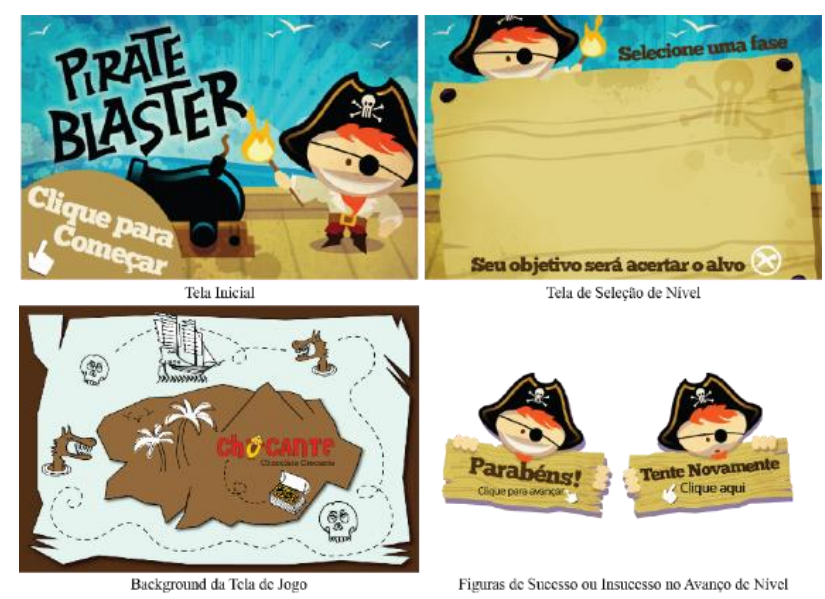

Figura 2 - Elementos gráficos do jogo "Pirate Blaster" Fonte: Elaborado pelos autores.

\subsubsection{Jogo com temática de marca (advergame)}

O jogo com temática de marca, representando a estratégia de advergame, foi chamado de Chocante - O Chocolate Crocante em Uma Aventura Pirata. Todos os elementos gráficos remetem à marca fictícia criada para este artigo, em suas cores, personagens e outros elementos presentes, como no baú com chocolates representando o tesouro pirata. O Grupo Experimental 2 interagiu com este jogo durante o experimento. A Figura 3 apresenta os elementos gráficos do advergame da marca fictícia Chocante Chocolate Crocante.

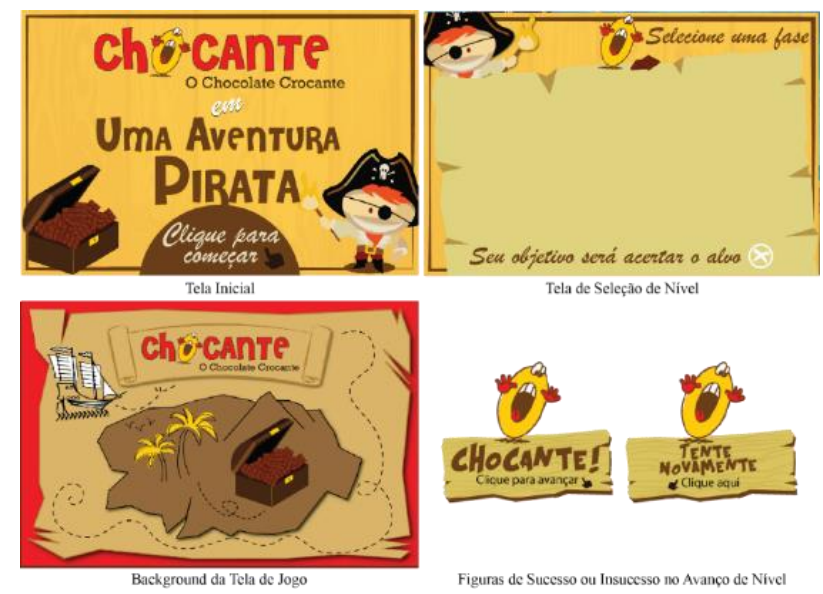

Figura 3 - Elementos gráficos do jogo "Chocante - O Chocolate Crocante em Uma Aventura Pirata" Fonte: Elaborado pelos autores.

\subsection{Variáveis dependentes}

\subsubsection{Atitude em relação à marca}

Assim como nos experimentos realizados por Cauberghe e Pelsmacker (2010) e Redondo (2010) a atitude em relação à marca foi utilizada para verificar a efetividade das estratégias de advergaming. De acordo com Keller (1993), a atitude em relação à marca é a avaliação da marca por parte dos consumidores.

A escala utilizada para medir a atitude em relação à marca foi formada por um conjunto selecionado dos itens apresentados por Bruner II (2009, p. 162). O autor exibe uma coleção de itens bipolares para medição da atitude em relação a marca, sendo que cada um desses estudos utilizou um conjunto distinto de itens. Bruner II (2009, p. 162) recomenda a utilização de uma escala de 
diferencial semântico, tipo Likert de 7 pontos. Nesse sentido, de acordo com os propósitos do estudo, os itens selecionados para medir a atitude em relação à marca, conforme apresentados por Bruner II (2009, p. 162) foram: ruim/boa; negativa/positiva; comum/diferenciada; não atraente/atraente; entediante/interessante; não divertida/divertida; e estática/dinâmica. Em adição, foram acrescentados os itens: ultrapassada/moderna; não interativa/interativa, baseado em Leng, Quah e Zainuddin (2010).

\subsubsection{Lembrança e Recordação}

Assim como foi realizado nos experimentos de Cauberghe e Pelsmacker (2010) e de Leng, Quah e Zainuddin (2010), a lembrança (recall) da marca também é uma variável dependente deste estudo. A consciência da marca é a presença dela na mente dos consumidores (AAKER, 2007). Segundo Aaker (2007), a consciência de uma marca é medida de acordo com as diferentes formas como os consumidores se recordam dela, variando desde o reconhecimento, passando pela lembrança (recall) e chegando ao top of mind.

Primeiramente, os participantes foram indagados se recordavam alguma marca presente no jogo. Em caso de resposta positiva, eram questionados se conseguiam recordar espontaneamente o nome dessa marca, conforme Ortega-Ruiz e Velandia-Morales (2011).

Em seguida, foram apresentados aos participantes cinco logotipos de diferentes marcas: três logos de marcas fictícias, a marca fictícia criada para este estudo e uma marca real, para medir a memória induzida, segundo Ortega-Ruiz e VelandiaMorales (2011).

\subsection{Procedimentos}

Para a realização correta do design elaborado para o experimento, era importante ter controle sobre aspectos como aleatorização dos grupos, tempo de jogo de cada participante e perfeito funcionamento do jogo no dispositivo em que se estivesse acessando o questionário.

O procedimento experimental foi $o$ seguinte: a) os sujeitos experimentais receberam email de convite para responder o questionário; b) os sujeitos experimentais participantes assinalaram, caso positivo, que estavam respondendo de um navegador de desktop ou notebook, para perfeito funcionamento do questionário; c) os sujeitos experimentais responderam a perguntas sobre a atitude em relação à marca, para as marcas Serenata de Amor e Arcor ao Leite (marcas reais para mascarar objetivos do estudo) e para a marca fictícia Chocante - Chocolate Crocante; d) os sujeitos experimentais tiveram que jogar um jogo (que foi randomizado de acordo com o grupo a que foram designados) durante três minutos; e) os participantes responderam novas perguntas sobre a atitude em relação à marca, para as marcas Arcor Bon o Bon e Arcor ao Leite (marcas reais, para mascarar objetivos do estudo) e para a marca fictícia Chocante - Chocolate Crocante; f) os participantes responderam a pergunta se lembravam de alguma marca presente no jogo; g) os participantes que assinalaram que se lembrava de alguma marca eram convidados a dizer qual era o nome dessa marca; $h$ ) os participantes eram perguntados se recordavam de qual das três marcas fictícias, além da marca fictícia Chocante - Chocolate Crocante, e uma marca real, se havia alguma delas presentes no jogo jogado, sendo que havia a opção de assinalar que não havia nenhuma marca presente no jogo.

\section{ANÁLISE E DISCUSSÃo DOS RESULTADOS}

Inicialmente, procedeu-se a uma inspeção por observação direta dos dados, para a verificação de outliers. Foram detectadas seis respostas em que o respondente havia assinalado somente uma única opção em todas as variáveis. Tais casos foram entendidos como outliers, sendo eliminados. Os casos estavam igualmente distribuídos entre os grupos (dois casos em cada grupo). Posteriormente, foi realizada a análise de outliers por meio da verificação de pontos extremos (maiores que Q3 + $3 *$ Dist. Interquartil, e menores que Q1 - 3* Dist. Interquartil) no gráfico de Box-Plot por variável, conforme indica Marôco (2003), sendo que outros valores não foram considerados como aberrantes e aceitos como inerentes à população, não tendo sido excluídos.

Conforme indicado por Dancey e Reidy (2006), verificou-se a normalidade dos dados a partir da inspeção visual de histogramas com a distribuição normal sobreposta e teste de normalidade, tendo a confirmação dessa garantia para a continuidade da análise dos dados e o procedimento das rotinas estatísticas selecionadas.

\subsection{Características da amostra}

A amostragem escolhida foi a amostragem não probabilística, em que alunos participaram voluntariamente (MALHOTRA, 2001). O uso de estudantes é recomendado para pesquisas envolvendo jogos (REDONDO, 2010), já que a maioria dos estudantes está habituada a esse meio de entretenimento. Além disso, diversos outros trabalhos relevantes nesta área, que realizaram experimentos, utilizaram amostras de graduandos, 
como: Nelson (2002), Leng, Quah e Zainuddin (2010), Wise et al. (2008) e Hernandez et al. (2004). A amostra foi composta por 165 estudantes de graduação, divididos equitativamente e de forma aleatória nos três grupos do experimento, apurandose 103 mulheres e 62 homens. A diferença no número de respondentes de cada sexo não foi proposital e não era objetivo do estudo coletar dados de uma quantidade similar de sujeitos por gênero.

\subsection{Atitude em relação à marca}

Após a realização da análise fatorial exploratória, este artigo objetivou utilizar os escores das variáveis remanescentes que compuseram os construtos para a realização de rotinas estatísticas de modo a comparar as médias, como forma de avançar no teste das hipóteses desse artigo. Optou-se por utilizar um método não refinado para computar as variáveis, o que facilita a interpretação dos dados, seguindo recomendações de DiStefano, Zhu e Mîndrilă (2009). De acordo com os autores, o cálculo da média das variáveis por unidade de análise é adequado quando o intuito é preservar as variações dos dados originais e, principalmente, não diferenciar as variáveis de acordo com o seu fator de carregamento (DiSTEFANO; ZHU; MÎNDRILĂ, 2009).

\subsubsection{Amostras pareadas (intra-grupos)}

Para a análise das condições antes e depois de cada grupo, a estatística escolhida foi o teste $t$ pareado. De acordo com Dancey e Reidy (2006), o teste $t$ pareado é usado quando os mesmos participantes fazem parte de ambas as condições.

$\mathrm{O}$ teste $t$ para amostras pareadas contribuiu para a decisão de suportar ou rejeitar as seguintes hipóteses desta artigo:

- $\mathrm{H}_{1}$ : Advergames e in-game advertisings provocam mudança significativamente positiva na atitude em relação à marca.

- $\mathrm{H}_{2}$ : Advergames provocam maior efeito na mudança significativamente positiva na atitude em relação à marca em comparação com os in-game advertisings.

A Tabela 2 apresenta o resultado do teste $t$ para amostras pareadas.

Tabela 2 - Teste $t$ para amostras pareadas

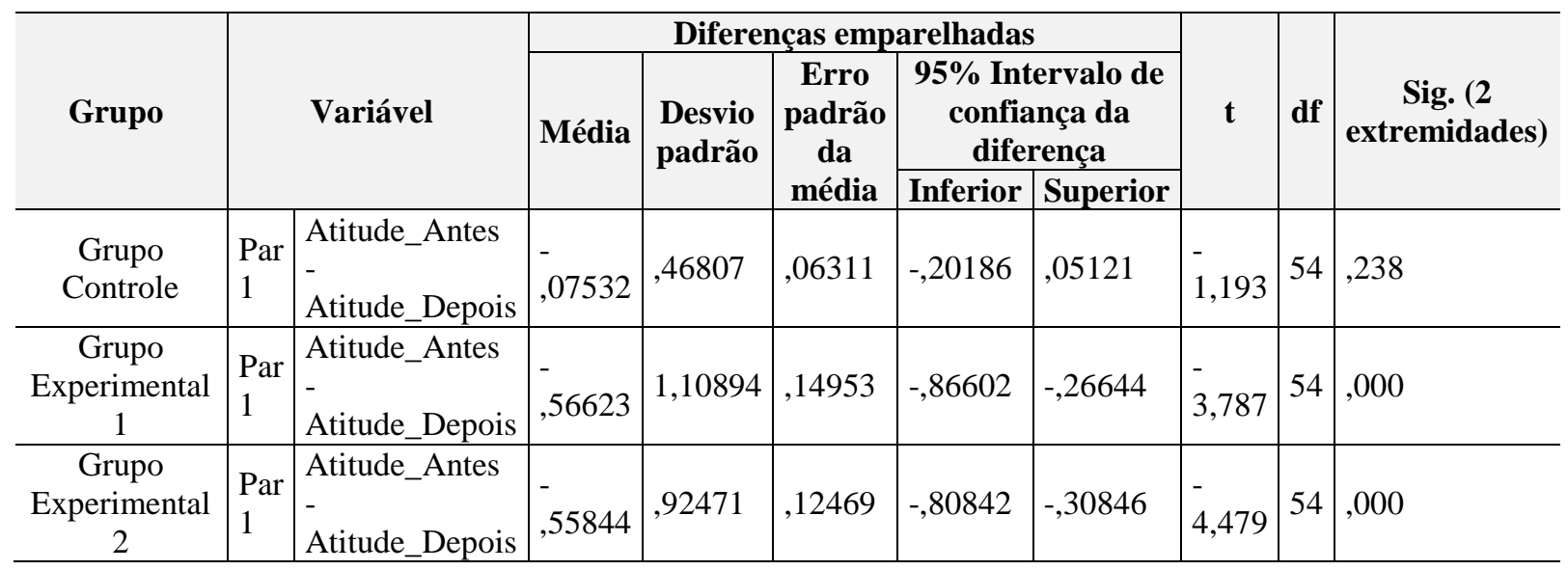

Fonte: Elaborado pelos autores.

Para o grupo Controle era esperado que não houvesse diferenças significativas entre as médias na condição antes e depois, sendo o resultado do teste $t(54)=-1,19, p>0,05$. Tal resultado era muito importante para eliminar a possibilidade de que a simples repetição da escala para a marca fictícia e o ato de jogar um jogo sem inserção de marca não iriam alterar a atitude dos respondentes. O tamanho do efeito foi calculado com base em Dancey e Reidy (2006, p. 222), subtraindo a média das condições e dividindo-a pela média dos desvios, chegando ao valor de $d=0,07$. Como mostra a Tabela 3 , este é um valor não relevante, conforme o esperado, já que não houve diferença significativa entre as médias. 
Tabela 3 - Interpretação do tamanho do efeito para teste $t$

\begin{tabular}{c|c|c}
\hline $\begin{array}{c}\text { Tamanho do } \\
\text { Efeito }\end{array}$ & $\boldsymbol{d}$ & $\begin{array}{c}\text { Percentagem de sobreposição } \\
(\boldsymbol{\%})\end{array}$ \\
\hline Pequeno & 0,2 & 85 \\
\hline Médio & 0,5 & 67 \\
\hline Grande & 0,8 & 53 \\
\hline
\end{tabular}

Fonte: Dancey e Reidy (2006, p. 222).

Para o grupo Experimental 1, que jogou o in-game advertising (jogo com inserção de marca no background), foi possível constatar diferenças significativas entre as médias antes e depois da interação com o jogo, $t(54)=-3,79, p<0,05$. Neste caso, $d=0,56$ indica um tamanho do efeito médio. Este resultado mostra que a interação com o in-game advertising foi efetiva em proporcionar um efeito positivo, na direção esperada previamente, no construto atitude em relação à marca. Estes resultados confirmam as afirmações realizadas por Hernandez et al. (2004), Redondo (2010) e Cauberghe e Pelsmacker (2010) de que a utilização de jogos para a inserção de marcas pode proporcionar tal incremento na atitude dos indivíduos em relação a uma marca.
Para o grupo Experimental 2, que jogou o advergame (jogo com temática de marca), foi possível constatar diferenças significativas entre as médias antes e depois da interação com o jogo, $t$ (54) $=-4,48, p<0,05$. Neste caso, $d=0,54$, indicando também um tamanho do efeito médio. Assim, é possível afirmar que estes resultados também confirmam as afirmações realizadas por Hernandez et al. (2004), Redondo (2010) e Cauberghe e Pelsmacker (2010) de que a utilização de jogos para inserção de marcas pode proporcionar tal incremento na atitude dos indivíduos em relação a uma marca.

$\mathrm{O}$ teste $t$ apontou diferenças significativas entre os grupos experimentais. O Gráfico 1 representa o efeito proporcionado pela variável dependente na variável independente.

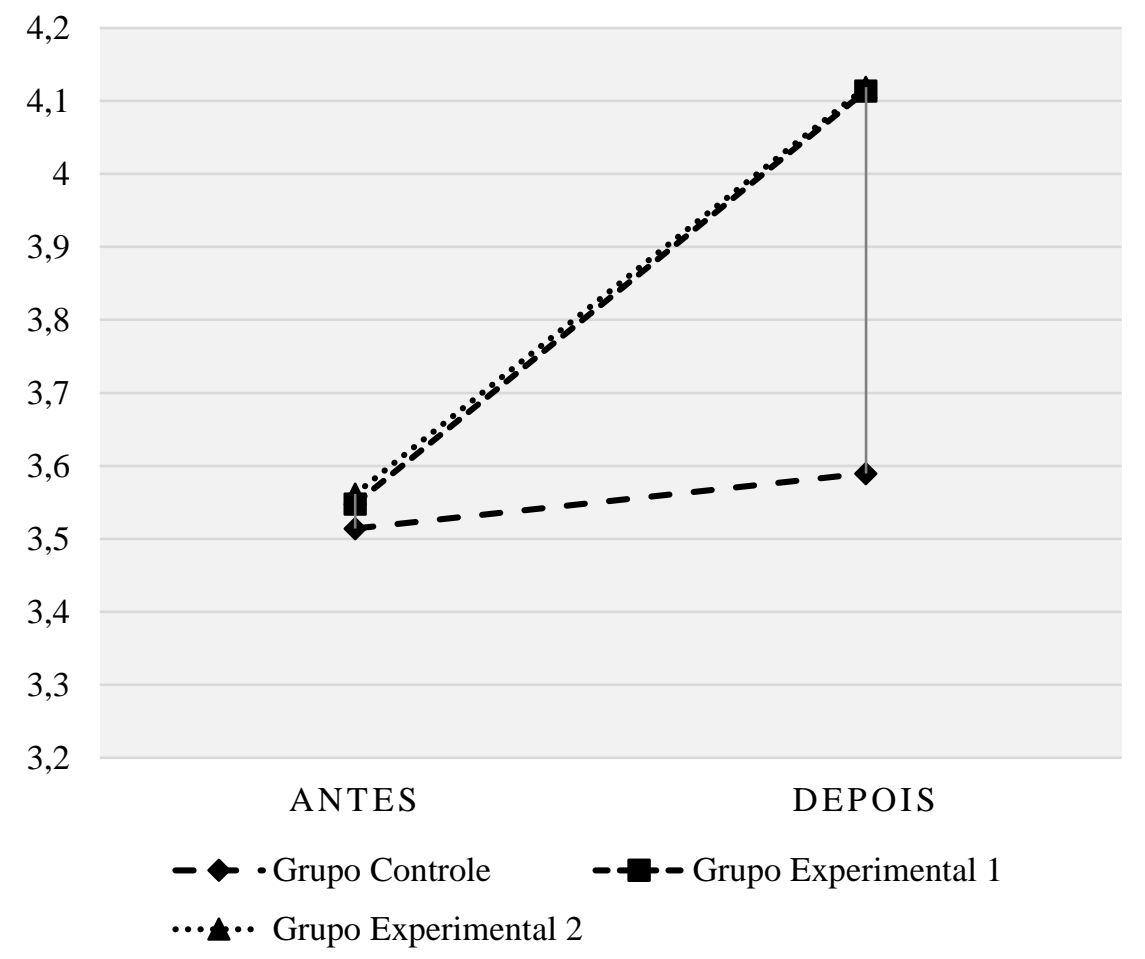

Gráfico 1 - Comparação entre médias da atitude em relação à marca Fonte: Elaborado pelos autores. 
Assim, o teste $t$ para amostras pareadas e o tamanho do efeito constatado dão fortes indícios de que a seguinte hipótese foi suportada:

- $\mathrm{H}_{1}$ : Advergames e in-game advertisings provocam mudança significativamente positiva na atitude em relação à marca.

Tal hipótese será constada após a realização da comparação de médias para amostras independentes. No entanto, os resultados indicam que a seguinte hipótese deve ser rejeitada:

- $\mathrm{H}_{2}$ : Advergames provocam maior efeito na mudança significativamente positiva na atitude em relação à marca em comparação com os in-game advertisings.

Esta hipótese também somente será rejeitada ou suportada parcialmente a partir da realização da comparação de médias para amostras independentes.

\subsubsection{Amostras independentes (entre grupos)}

Para verificar a diferença no efeito da variável independente na variável dependente, comparando os grupos, calculou-se uma nova variável, a partir da subtração da média da condição antes da média da condição depois. Esse cálculo permite avaliar diretamente o efeito causado em cada grupo de sujeitos experimentais.

Para tal, recomenda-se utilizar a ANOVA, que procura verificar se existem diferenças nas médias dos grupos, determinando a média geral e verificando o quão diferente cada média individual é da média geral (DANCEY; REIDY, 2006). Conforme recomendam Dancey e Reidy (2006, p. 311 ), é preciso que o valor do teste de igualdade das variâncias de Levene seja superior a 0,05. No entanto, o valor calculado esteve abaixo deste limite.

Assim, como recomendam Dancey e Reidy (2006), optou-se pela realização do teste KruskalWallis, equivalente não paramétrico da ANOVA, utilizado quando os dados não satisfazem as condições requeridas para uma ANOVA paramétrica.

A Tabela 4 apresenta as estatísticas do teste.

Tabela 4 - Kruskal-Wallis

\begin{tabular}{l|l}
\hline \multicolumn{1}{c|}{ Teste } & \multicolumn{1}{|c}{ Diferença_Ch1 } \\
Q_
\end{tabular}

Fonte: Elaborado pelos autores.

Os resultados apresentados na Tabela 4 mostram que $\chi 2=9,99$, com nível de significância alcançado de $p<0,05$. Dessa forma, é possível afirmar que pelo menos um dos grupos tem a média significativamente diferente dos demais. Assim, a análise post-hoc pode ser realizada com o intuito de verificar diferenças emparelhadas.
Diferente da ANOVA não existem testes que seguem naturalmente o Kruskal-Wallis. Assim, é recomendado fazer as comparações usando o teste de Mann-Whitney (DANCEY; REIDY, 2006). A primeira comparação se dá entre o grupo Controle e o grupo Experimental 1. A Tabela 5 apresenta as estatísticas do teste.

Tabela 5 - Teste de Mann-Whitney 1

\begin{tabular}{l|l}
\hline \multicolumn{1}{c|}{ Teste } & \multicolumn{1}{c}{ Diferença_Ch } \\
\hline Mann-Whitney U & 1079,000 \\
\hline Wilcoxon W & 2619,000 \\
\hline Z & $-2,649$ \\
\hline Sig. Assint. (2 caudas) &, 008 \\
\hline
\end{tabular}

Fonte: Elaborado pelos autores.

Os resultados apresentados na Tabela 4 mostram que $Z$ é $-2,65$, com nível de significância alcançado de $p=0,008$. Dessa forma, é possível afirmar que a média das diferenças do grupo Experimental 1 é maior que a do grupo Controle, na direção esperada, conforme mostra a Tabela 4. 
Além da constatação de que há diferença entre os grupos, na direção esperada, o tamanho do efeito foi calculado de acordo com recomendações de Field (2013), dividindo o valor $Z$ pela raiz quadrada do número total de observações. Assim, o tamanho do efeito é $r=0,25$. A Tabela 6 apresenta como interpretar o tamanho do efeito para este teste.

Tabela 6 - Tamanho do efeito

\begin{tabular}{c|l|c}
\hline $\begin{array}{c}\text { Efeito } \\
\text { Pequeno }\end{array}$ & Efeito Médio & $\begin{array}{c}\text { Grande } \\
\text { Efeito }\end{array}$ \\
\hline 0.10 & 0.30 & 0.50 \\
\hline
\end{tabular}

Fonte: Adaptado de Field (2013).

Como mostra a Tabela 6, o tamanho do efeito de $r=0,25$ pode ser interpretado como pequeno-médio, mais próximo da condição de médio efeito.
A segunda comparação se dá entre o grupo Controle e o grupo Experimental 2. A Tabela 7 apresenta as estatísticas do teste.

Tabela 7 - Teste de Mann-Whitney 2

\begin{tabular}{l|l}
\hline \multicolumn{1}{c|}{ Teste } & \multicolumn{1}{c}{ Diferença_Ch } \\
\hline Mann-Whitney U & 1047,500 \\
\hline Wilcoxon W & 2587,500 \\
\hline Z & $-2,811$ \\
\hline Sig. Assint. (2 caudas) &, 005 \\
\hline
\end{tabular}

Fonte: Elaborado pelos autores.

Os resultados apresentados na Tabela 7 mostram que $Z=-2,81$, com nível de significância alcançado de $p=0,005$. Dessa forma, é possível afirmar que a média das diferenças do grupo Experimental 2 é maior que a do grupo Controle, na direção esperada, conforme mostra a Tabela 6 .

Além da constatação de que há diferença entre os grupos, na direção esperada, o tamanho do efeito foi calculado com base nas recomendações de Field (2013), sendo o resultado de $r=0,27$. O tamanho do efeito de $r=0,27$ pode ser interpretado como pequeno-médio, mais próximo da condição de médio efeito.

A terceira comparação se dá entre o grupo Experimental 1 e o grupo Experimental 2. A Tabela 8 apresenta as estatísticas do teste.

Tabela 8 - Teste de Mann-Whitney 3

\begin{tabular}{l|l}
\hline \multicolumn{1}{c|}{ Teste } & \multicolumn{1}{c}{ Diferença_Ch } \\
\hline Mann-Whitney U & 1443,000 \\
\hline Wilcoxon W & 2983,000 \\
\hline Z &,- 418 \\
\hline Sig. Assint. (2 caudas) &, 676 \\
\hline
\end{tabular}

Fonte: Elaborado pelos autores.

Os resultados apresentados na Tabela 8 mostram que $Z=-0,42$, com nível de significância alcançado de $p=0,676$. Dessa forma, é possível afirmar que não há diferença significativa entre os grupos testados. O tamanho do efeito de $r=0,04$ reflete um efeito interpretado como sendo nulo.

Os resultados mostram que as estratégias de advergaming proporcionaram mudanças positivas em relação ao construto atitude em relação à marca, confirmando as afirmações de Hernandez et al.
(2004), Redondo (2010) e Cauberghe e Pelsmacker (2010) de que a utilização de jogos para a inserção de marcas pode proporcionar tal incremento na atitude dos indivíduos em relação a uma marca. Os resultados confirmam a seguinte hipótese do estudo:

- $\mathrm{H}_{1}$ : Advergames e in-game advertisings provocam mudança significativamente positiva na atitude em relação à marca. 
Contudo, os resultados desta análise não corroboram as afirmações feitas por Leng; Quah; Zainuddin (2010), Wise et al. (2008), Oliveira (2012), Parreño; Pérez; Garcia (2010), Hernandez et al. (2004), Redondo (2010), Cauberghe; Pelsmacker (2010) quando afirmam que o advergame é superior ao in-game advertising no efeito em relação ao construto atitude em relação à marca. Assim, a seguinte hipótese deste estudo é rejeitada:

- $\mathrm{H}_{2}$ : Advergames provocam maior efeito na mudança significativamente positiva na atitude em relação à marca em comparação com os in-game advertisings.

Dessa forma, é possível afirmar que as duas estratégias de exposição de marcas utilizando jogos podem ser efetivas na mesma proporção quando o intuito é provocar melhora na atitude em relação à marca. Assim, o contexto e a qualidade da inserção, juntamente com o objetivo da estratégia promocional da empresa é que irão determinar qual dessas estratégias é mais adequada em cada situação.

No entanto, como se verá adiante, a lembrança e a recordação podem apresentar diferentes resultados na comparação entre as duas estratégias de advergaming, podendo determinar qual delas é a melhor opção para a empresa, de acordo com o nível de conscientização da marca de seus clientes.

\subsection{Lembrança e recordação}

De acordo com Kotler e Keller (2012), a conscientização da marca reflete a capacidade do consumidor em lembrar ou reconhecer uma marca dentro de uma categoria. Segundo os autores, a lembrança é particularmente importante fora do ambiente de compra e consumo, enquanto a recordação representa um importante papel durante o momento de seleção de alternativas no ponto de venda. Assim, o experimento buscou avaliar se os participantes se lembravam espontaneamente da marca presente no jogo e, depois, se, dentre outras marcas, os mesmos eram capazes de reconhecer a marca que estava presente no jogo.

As análises realizadas neste item permitiram testar a seguinte hipótese deste estudo:

- $\mathrm{H}_{3}$ : As frequências observadas de lembrança e recordação da marca presente no jogo são significativamente maiores em advergames em comparação a in-game advertisings.

A pergunta realizada inicialmente foi: "Você se lembra de alguma marca presente no jogo em que jogou?". Os participantes tinham a opção de marcar "Sim" ou "Não". Em caso de terem assinalado "Sim", eram perguntados: "Você consegue dizer qual era o nome dessa marca?". As respostas foram classificadas da seguinte maneira: "Não lembrou", quando o participante assinalou "Não" na primeira pergunta ou quando deixou o campo em branco na segunda resposta; "Confundiuse", quando citou o nome de alguma outra marca que não a marca fictícia criada para o jogo; "Lembrou parcialmente", quando a resposta do participante conteve alguma parte do nome da marca fictícia que não o seu nome principal "Chocante" (por exemplo, "Crocante", "Chocolate Crocante"); e "Lembrou", quando a resposta conteve a palavra "Chocante".

O Gráfico 2 apresenta de maneira visual os resultados de frequência de lembrança da marca para os grupos Controle, grupo Experimental 1 e grupo Experimental 2. 


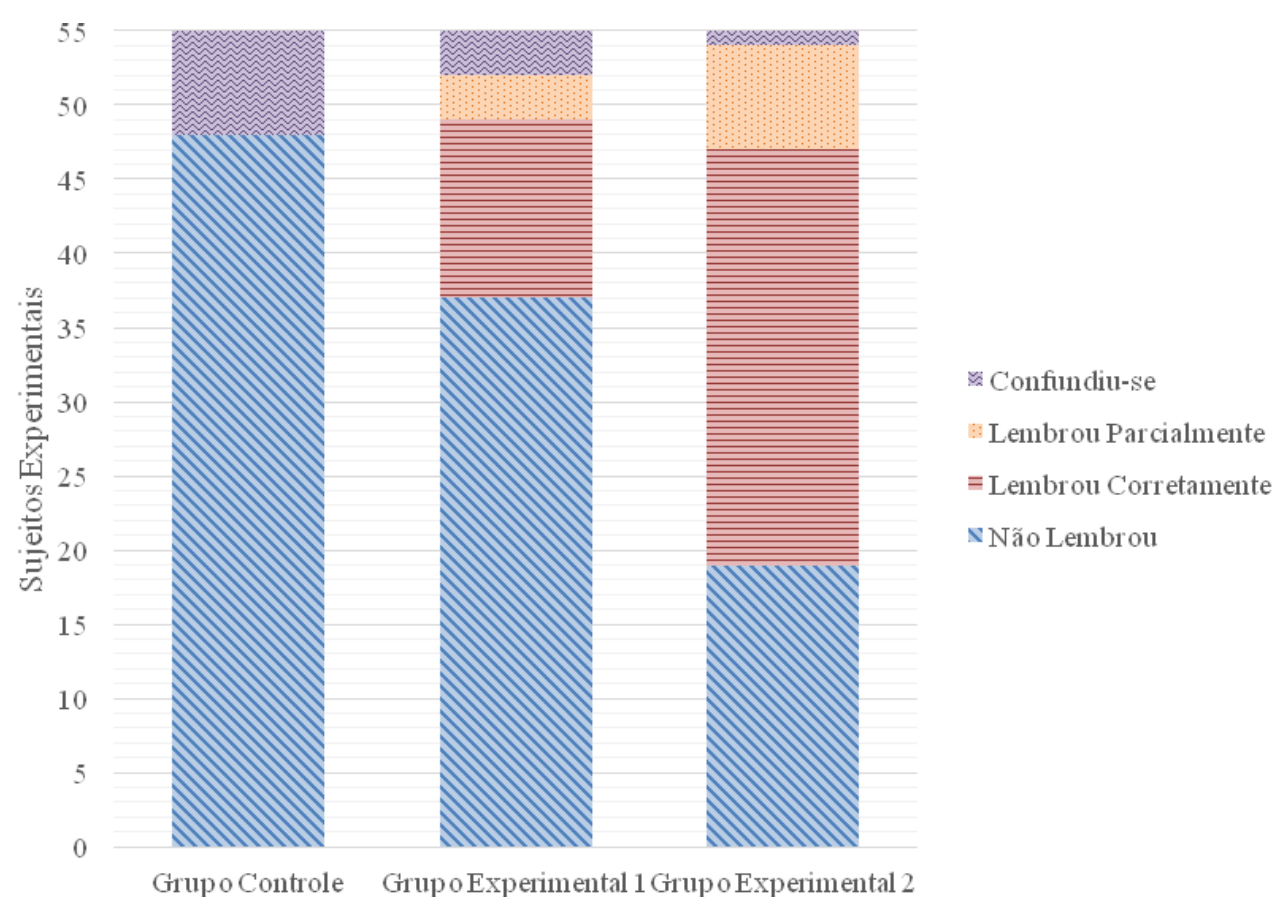

Gráfico 2 - Frequência de lembrança espontânea da marca - grupo Controle, grupo Experimental 1 e grupo Experimental 2

Fonte: Elaborado pelos autores.

O resultado endossa a validade da seguinte hipótese: "As frequências observadas de lembrança e recordação da marca presente no jogo são significativamente maiores em advergames em comparação à in-game advertisings". Esses resultados são semelhantes aos achados de Leng, Quah e Zainuddin (2010) e Cauberghe e Pelsmacker (2010). Lembrança superior para a interação com o advergame pode ser atribuída à natureza dessa estratégia de comunicação, criada em torno do universo da marca, consequentemente levando à sua muito maior exposição e interação com usuários do que as observadas no in-game advertising, em que a marca apenas é exposta como elemento secundário no jogo.

Apresentam-se a seguir os resultados de recordação, que, segundo Keller (1993), refletem a identificação correta da marca determinada pela categoria do produto ou algum outro tipo de sugestão. Conforme descrito no item 4.2.2, os participantes responderam à seguinte pergunta: "É possível que no jogo que você jogou havia alguma marca presente. Você consegue se recordar de qual dessas marcas seria? ". Foram apresentadas graficamente cinco marcas, sendo três marcas fictícias, criadas para esta questão (Chocolante, MilkMu e Latte), a marca fictícia criada para este estudo (Chocante - Chocolate Crocante) e uma marca real (Arcor), havia, também, a opção de assinalar "Não havia nenhuma marca presente".

O Gráfico 3 apresenta de maneira visual os resultados de frequência de recordação da marca presente no jogo n os grupos Controle, Grupo Experimental 1 e Grupo Experimental 2. 


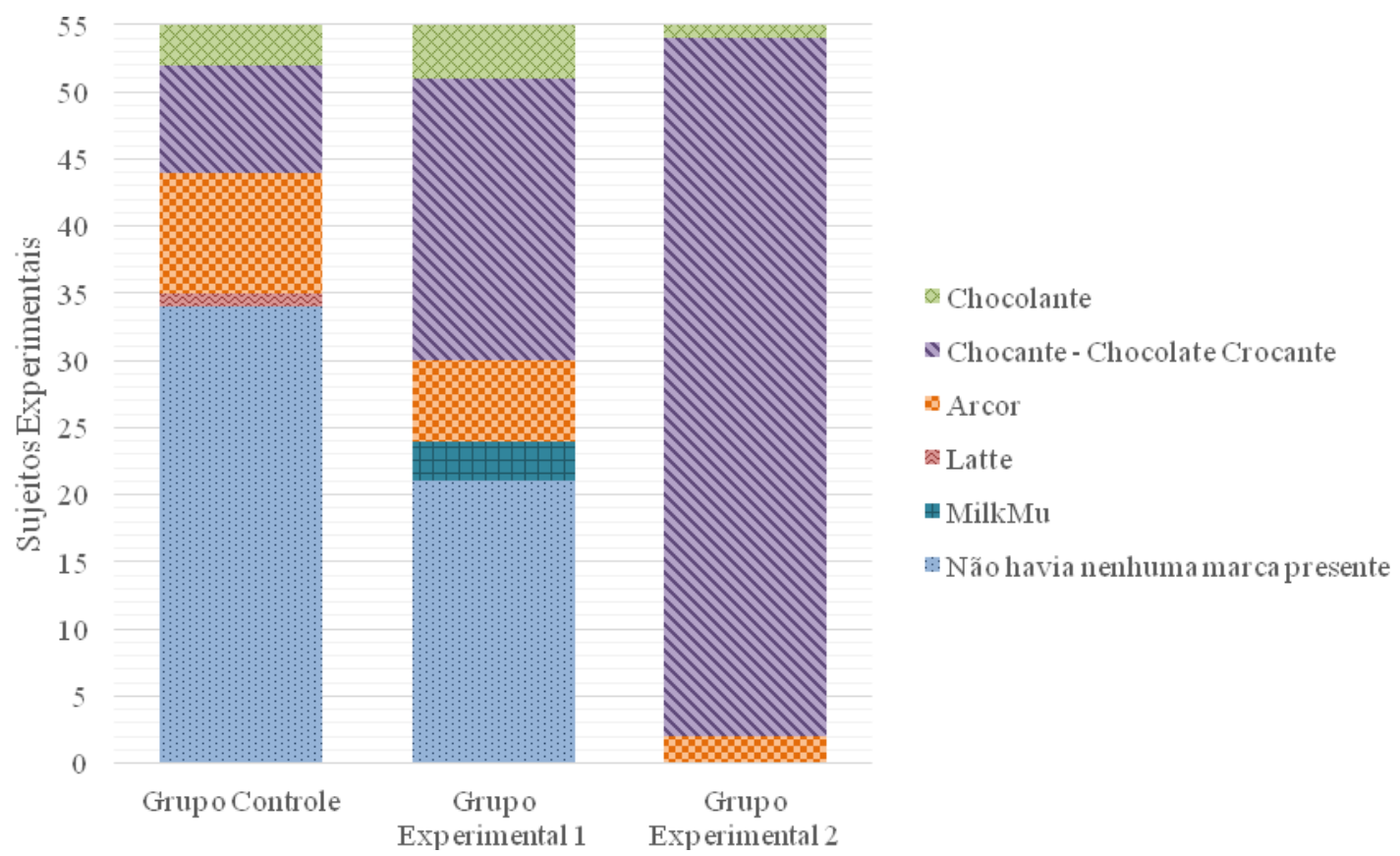

Gráfico 3 - Frequência de recordação de marca - grupo Controle, grupo Experimental 1 e grupo Experimental 2 Fonte: Elaborado pelos autores.

Para verificar a distribuição de frequência das respostas é apresentada a tabulação cruzada, de acordo com Malhotra (2001), resulta em tabelas que refletem a contribuição conjunta de duas ou mais variáveis com um número limitado de categorias. Neste caso, as outras marcas apresentadas foram concatenadas em uma mesma categoria com o intuito de realizar o teste de diferença a partir de médias e proporções, conforme indica Malhotra (2001). A Tabela 9 apresenta a tabulação cruzada da recordação onde foram agrupadas as respostas por marcas ou a percepção de que não havia marcas presentes no jogo.

Tabela 9 - Tabulação Cruzada da Recordação

\begin{tabular}{c|c|c|c|c}
\hline Marcas & $\begin{array}{c}\text { Grupo } \\
\text { Controle }\end{array}$ & $\begin{array}{c}\text { Grupo } \\
\text { Experimental 1 }\end{array}$ & $\begin{array}{c}\text { Grupo } \\
\text { Experimental 2 }\end{array}$ & Total \\
\hline Chocante - Chocolate Crocante & $8_{\mathrm{a}}$ & $21_{\mathrm{b}}$ & $52_{\mathrm{c}}$ & 81 \\
\hline Outras Marcas & $13_{\mathrm{a}}$ & $13_{\mathrm{a}}$ & $3 \mathrm{~b}_{\mathrm{b}}$ & 29 \\
\hline Não havia nenhuma marca presente & $34_{\mathrm{a}}$ & $21_{\mathrm{b}}$ & 0 & 55 \\
\hline Total & 55 & 55 & 55 & \\
\hline
\end{tabular}

Fonte: Elaborado pelos autores.

Na Tabela 9 os valores na mesma linha e subtabela que não compartilham o mesmo subscrito são bastante diferentes em $\mathrm{p}<.05$ no teste de igualdade bilateral para proporções de coluna. As células sem nenhum subscrito não são incluídas no teste. Os testes consideram variâncias iguais. Assim, é possível observar que as frequências observadas em relação à marca fictícia Chocante - Chocolate Crocante diferem entre si; para outras marcas os grupos Controle e Experimental 1 não diferem entre si, mas do grupo Experimental 2; e para a percepção dos sujeitos de que não havia marca no jogo os grupos Controle e Experimental 1 diferem entre si. $\mathrm{O}$ teste de qui-quadrado de Pearson foi significante ao nível de $95 \%$.

Assim, pode-se, definitivamente, confirmar a seguinte hipótese:

$\mathrm{H}_{3}$ : As frequências observadas de lembrança e recordação da marca presente no jogo são 
significativamente maiores em advergames em comparação à in-game advertisings.

Este resultado é semelhante aos achados de Leng, Quah e Zainuddin (2010) e Cauberghe e Pelsmacker (2010).

Dessa forma, apesar de a hipótese $\mathrm{H}_{2}$ ter sido rejeitada, mostrando que o advergame provoca o mesmo efeito positivo na atitude em relação à marca que o in-game advertising, esta estratégia se mostrou superior ao elevar a frequência de lembrança e recordação dos sujeitos, apontando uma vantagem para esta estratégia neste sentido.

\section{CONCLUSÕES}

Como o número de publicações científicas que exploram o tema pesquisado é ainda incipiente, este artigo buscou contribuir para a compreensão da efetividade do advergaming, explorando, principalmente, as características distintas de suas duas principais estratégias: advergames e in-game advertisings. A utilização do experimento foi fundamental para comparar a efetividade das duas estratégias, na medida em que o controle do nível de exposição da marca na variável independente permitiu constatar os efeitos proporcionados na medição das variáveis dependentes.

A primeira contribuição dos achados deste artigo consistiu em apresentar um cenário onde a atitude em relação à marca é positivamente impactada, de maneira significativa, pela recente interação com um jogo com a inserção de uma marca. Estes resultados apontam para uma perspectiva de que a utilização de jogos para a inserção de marcas seja, de fato, uma importante estratégia para a promoção de marcas, alcançando os resultados esperados na avaliação da marca com relação à capacidade comprovada de atender a uma necessidade relevante. Conforme argumentam Jaffe (2008) e Parreño, Pérez e Garcia (2010), a utilização de jogos para a inserção de marcas é apontada como uma alternativa viável para a saturação da exposição de mensagens a que os consumidores estão sujeitos, em que atuam os processos de atenção seletiva, distorção seletiva e retenção seletiva.

Os achados do artigo também mostraram que não houve diferenças significativas entre este impacto positivo proporcionado por advergames quando comparados à in-game advertising. Isso mostra que o mais importante é que nestas estratégias a mensagem seja interativa e possa promover maior envolvimento, dependendo apenas da qualidade do entretenimento proporcionado pelo jogo, seu tema e sua narrativa (JAFFE, 2008). Assim, as duas estratégias podem ser efetivas para impactarem a atitude em relação à marca em igual medida, dependendo do quão assertivo tenham sido planejamento e a execução desta estratégia promocional pela empresa.

Os resultados em relação à lembrança e recordação apresentaram maior efetividade na interação com o advergame. Como era o esperado, a interação com o jogo com a temática da marca fortalece bastante o nível de conscientização de marca, que, conforme colocam Kotler e Keller (2012), são muito importantes para a formação da intenção de compra, dentro e fora do ambiente de consumo. Assim, conforme sustentam Parreño, Pérez e Garcia (2010), a utilização de jogos par a inserção de marcas reforça o reconhecimento da marca, a familiaridade com a marca e a identificação com a marca.

Este artigo conclui que a utilização do advergaming como estratégia promocional é capaz de encontrar consumidores mais receptivos às mensagens de marca, sendo também efetiva na construção de uma atitude favorável em relação a ela, assim como no aspecto da construção da conscientização de marca. De uma perspectiva mais ampla, este artigo contribui para a compreensão do comportamento do consumidor mediante a utilização de estratégias promocionais interativas, mostrando que esta adaptação/evolução da forma como se alcançam os consumidores não somente é necessária, como também interativa no cenário atual de disseminação irrevogável da internet e fragmentação de mídias.

Este trabalho tem implicações gerenciais importantes para profissionais interessados em melhorar a atitude e a conscientização de marcas pelos consumidores, assim como para criadores de jogos, cujas receitas provêm dessas obras, a partir de negociação de espaços (in-game advertising) ou completa construção ou adaptação de jogos a marcas (advergames). $\mathrm{O}$ artigo mostra que tais estratégias interativas de fato funcionam e podem ser utilizadas na promoção de marcas, com intuito de estimular seu conhecimento, avaliação positiva e consumo. Os achados mostram que advergames e in-game advertising são efetivos em gerar um efeito positivo na atitude em relação à marca, conforme sustentam os estudos de Lamb, Hair e McDaniel (2010); Tuten e Solomon (2013); Jaffe (2008); Leng, Quah e Zainuddin (2010); Wise et al., (2008); Oliveira (2012); Parreño, Pérez e Garcia (2010); Hernandez et al (2010); Redondo (2010); Cauberghe e Pelsmacker (2012). Sendo que os advergames proporcionam maiores índices de lembrança e recordação da marca, conforme também verificado em trabalhos como os de Leng, Quah e Zainuddin (2010) e Cauberghe e Pelsmacker (2012).

Aponta-se como limitação principal do estudo o fato de ter sido realizado apenas um estudo experimental, o que não possibilitou testar os efeitos 
proporcionados em dispositivos móveis e consoles, apenas em desktops. À medida que estes são canais importantes para inclusão destas mensagens, há uma dificuldade de interpretar os resultados para tais canais.

Como sugestão para estudos futuros, podese apontar a realização de experimentos que comparem estratégias interativas diretamente com estratégias mais intrusivas e interruptivas em contextos reais, incluindo dispositivos móveis e consoles. Este trabalho buscou avaliar em maior grau a diferença entre as estratégias in-game advertising e advergame, como forma de aumentar a compreensão deste fenômeno e suas vertentes. No entanto, é possível ampliar o contexto de análise buscando comparar a utilização de jogos, enquanto variáveis independentes de um experimento, com formatos mais tradicionais de propaganda, isso permitiria maior aprofundamento da questão geral sobre a efetividade destas estratégias, contribuindo para um cenário em que o conhecimento acadêmico gerado na área de Marketing ajuda a esclarecer o comportamento do consumidor e a dinâmica de mercado, bem como o direcionamento e a alocação mais assertivos e seu controle em métricas dos orçamentos promocionais, contribuindo para melhorar o desempenho desta função e a filosofia organizacional.

\section{REFERÊNCIAS}

Aaker, David A. Construindo marcas fortes. São Paulo: Bookman, 2007.

Bruner II, Gordon C. Marketing scales handbook: a compilation of multi-item measures for consumer behavior \& advertising research. Volume 5. Carbondale: GCBII Productions, 2009.

Campi, Mônica. A vez dos advergames na internet. 2012. Disponível em: <

http://exame.abril.com.br/marketing/noticias/a-vezdos-advergames>. Acesso em 5 de Setembro de 2012.

Cauberghe, Verolien; Pelsmacker, Patrick. The impact of brand prominence and game repetition on brand responses. Journal of Advertising, v. 39, n.1, 2010.

Dancey, C. P; Reidy, J. Estatística sem matemática para psicologia. Porto Alegre: Artmed, 2006.

Distefano, C.; Zhu, M,; Mîndrilă, D. Understanding and Using Factor Scores: Considerations for the Applied Researcher. Practical Assessment,
Research \& Evaluation, Vol 14, No 20, Page 3, 2009.

Field, Andy. Discovering Statistics using IBM SPSS Statistics. Londres: SAGE Publications Ltd., 2013.

Gaarbo, Mark; Johsen, Johan. K. Generation game and how to reach them: a study of advergames affect on brand attitude and of gaming habits in a Danish context. 2012. Tese (Doutorado em Marketing). Copenhagen Business School.

Hernandez, Monica D.; Chapa, Sindy; Minor, Michael S.; Maldonado, Cecilia; Barranzuela, F. Hispanic attitudes toward advergames: a proposed model of their antecedents. Journal of Interactive Advertising, v. 5, n. 1, 2004, p. 74-83.

IBOPE. Game on: artigo publicado no meio \& mensagem do dia 10 de setembro. 2012. Disponível em <http://www.ibope.com.br/pt$\mathrm{br} /$ conhecimento/artigospapers/Paginas/Gameon.aspx>. Acesso em 28 de setembro de 2012.

Jaffe, Joseph. O declínio da mídia de massa. São Paulo: M. Books, 2008.

Keller, Kevin Lane. Conceptualizing, measuring, and managing customer-based brand equity. Journal of Marketing, v.57, p.1-22, 1993

Kotler, P; Keller, K. L. Administração de Marketing. 14. ed. São Paulo: Pearson PrenticeHall, 2012.

Lamb, C. W; Hair, J. F.; McDaniel, C. Marketing. Mason: Cengage Learning, 2010.

Leng, H. K; Quah, S. L.; Zainuddin, F. The Obama effect: an exploratory study on factores affecting brand recall in online games. International Journal of Trade, v. 1, n. 1, Junho, 2010.

Malhotra, N. K. Pesquisa de marketing: uma orientação aplicada. 3. ed. Porto Alegre: Bookman, 2001.

Marôco, J. Análise Estatística de dados - com utilização do SPSS, Edições Sílabo, Lisboa, pág. 36, 2003.

Nelson, M. R. Recall of brand placements in computer/vídeo-games. Journal of Advertising Research, Março/Abril, 2002, p. 60-92.

Nelson, M.R.; Keun, H.; Yaros, R. Advertainment or adcreep game player's attitudes toward advertising and product placements in computer 
games. Journal of Interactive Advertising, v. $5, \mathrm{n}^{\circ} 1$, 2004, p. 3-21.

Newzoo. 2016 Global Games Market Report: trends, insights \& projections. Disponível em $<$ https://newzoo.com/wpcontent/uploads/2016/01/N ewzoo_2016_Global_Games_Market_Report_Dum my.pdf $>$. Acesso em 20 de Setembro de 2017.

Oliveira, Sandra. Advergames: acção viral WoMo ("Word of Mouse"). Revista Estudos em Comunicação, Portugal, n. 11, p. 227-243, 2012.

Ortega-Ruiz, C. A.; Velandia-Morales, A. Influencia del advergaming y el advertising em la recordación y reconocimiento de una marca.

Revista Latino Americana de Psicologia, v. 43, n³, 2011, p.511-520.

Parreño, José; Pérez, Rafael; García, Isabel. Nuevas fórmulas publicitarias: lós advergames como herramienta de lãs comunicaciones de marketing. Cuadernos de Gestión, v. 12, n. 2, 2010, p. 43-58.

Redondo, Ignacio. The effectiveness of casual advergames o adolescents brand attitudes. European Journal of Marketing, v. 46, n. 11/12, 2010.
Söbke, H., Bröker, T. (2015). Um advergame para browser como catalisador da comunicação: tipos de comunicação em jogos de vídeo. Comunicação $E$ Sociedade, 2753-94. doi:10.17231/comsoc.27, 2015.

Tuten, Tracy; Solomon, Michael. Social Media Marketing. Pearson, 2013.

Vaz, Gabriel G. Estratégias da publicidade nos games de segunda geração: potencialização nas redes digitais. 2010. 76 f. Monografia (conclusão de curso de Publicidade e Propaganda) - Universidade Federal do Rio Grande do Sul, Porto Alegre, 2010.

Wang, L.; Lee, C.; Mantz, T. K.; Hung H. C. (2015). Effects of flow and self-construal on player perception of brand personality in advergames. Social Behavior \& Personality: An International Journal, 43(7), 1181-1192. doi:10.2224/sbp.2015.43.7.1181

Wise, Kevin; Bolls, Paul D.; Kim, Hyo; Venkataraman, Arun; Meyer, Ryan. Enjoyment of advergames and brand attitudes: the impact of thematic relevance. Journal of Interactive Advertising, v. 9, n. 1, 2008, p. 27-36. 